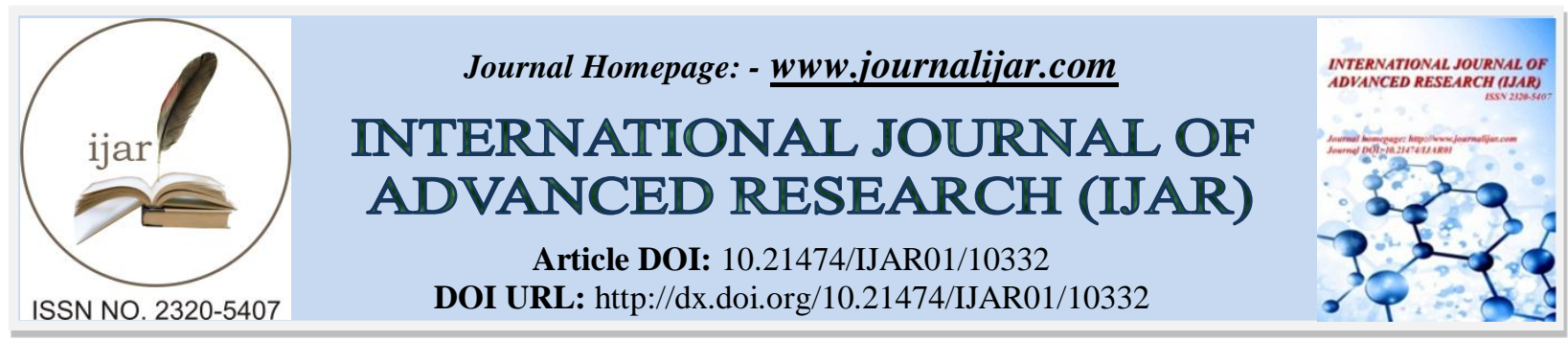

RESEARCH ARTICLE

\title{
ESTHETIC REHABILITATION OF SMILE CONJUGATED WITH FUNCTIONAL PRESERVATION OF ANTERIOR GUIDANCE - A CASE REPORT
}

\author{
Soumadip Niyogi ${ }^{1}$, Jayanta Bhattacharyya ${ }^{2}$, Manas Konar ${ }^{1}$ and Samiran Das ${ }^{3}$ \\ 1. MDS , Guru Nanak Institute Of Dental Sciences \& Research, Kolkata, 9/1, Mahendra Bose Lane Kolkata - \\ 700003. \\ 2. Principal (H.O.D), Professor, Guru Nanak Institute Of Dental Sciences \& Research, Kolkata, 9/1, Mahendra \\ Bose Lane Kolkata -700003. \\ 3. Professor, Guru Nanak Institute Of Dental Sciences \& Research, Kolkata, 9/1, Mahendra Bose Lane Kolkata - \\ 700003.
}

\section{Manuscript Info}

(...........................

Manuscript History

Received: 25 November 2019

Final Accepted: 27 December 2019

Published: January 2020

Key words:-

Anterior Guidance, Customized Guide

Table, Disclusion.Introduction

\section{Abstract}

Prosthetic rehabilitation should not only consider esthetic superiority but also encompass all components of an ideal occlusion that does not wear, promotes a healthy periodontium, and maintains a quiet neuromuscular mechanism. This case report illustrates the importance of preservation of the anterior guidance and the exemplary procedural method of achieving that while restoring the anterior teeth esthetically and safeguarding functionality amidst superfluous use of lavish software programs to simulate the same.

Copy Right, IJAR, 2020,. All rights reserved.

\section{Introduction:-}

A desire to look attractive is no longer a sign of ostentation. The aim is not only to achieve an aesthetic superiority but also consider all components of an ideal occlusion that does not wear, promotes a healthy periodontium ,temporomandibular joint, and maintains a quiet neuromuscular mechanism. ${ }^{1}$ An unfavourable anterior guidance often contributes to bone loss and mobility when there are vulnerable periodontal tissues and exceeding amount of forces, as it is the dynamic relationship of the lower and upper anterior teeth in all frames of motion and is deterrent in nature by providing disclusion in excursive movements.

Case Report:

A 39 year old female patient reported to the department of Prosthodontics and Crown \& Bridge with the complaint of unsightly appearance of her front teeth and displeasing appearance of her smile which seemed to have gradually increased over a period of time. After thorough corroboration of history and clinical examination, findings revealed loss of proximal contacts and architectural contour of the papilla, with slight amount of increased tonicity of the circumoral musculature which provided a strained appearance of the lower third of the face during smiling, periodontal health was non-contributory with sufficient width of the attached gingiva [Figures 1 and 2]. After further discussion and consent, rehabilitation of anterior teeth esthetically through customizing and preserving the anterior guidance was formulated keeping in mind the abiding functional harmonization.

Preliminary impressions were made with irreversible hydrocolloid impression material and diagnostic casts obtained. A face bow transfer was made and casts were articulated in a semi adjustable articulator with the help of 
centric relation records facilitated through an anterior deprogrammer, and articulator was subsequently programmed through protrusive records. The diagnostic casts were thoroughly evaluated and persisting with the micro and macro aesthetic considerations midline, symmetry and prolcinations were noted. The amount of the spacing present were calculated and judged according to the golden proportions and maintained to achieve the correct width to height ratio. A diagnostic wax-up was done to set up anterior guidance in unison with the patient's own incisal guidance and to create a template for making provisional restorations. This was achieved through fabrication of the customized guide table ( self-cure acrylic resin was manipulated in a dough stage and placed on to the guide table, the incisal end of the incisal pin was moved so as to simulate the excursive movements, process repeated till the resin had achieved its set and produced an unperturbed path without interferences $)^{2}$ thereby absolutely preserving patients own path of guidance [Figure 3]. Minor alterations of the length of the anterior teeth were achieved to provide desired amount of overjet and overbite and maintain the incisal edge positions to revert back the harmony of smile. Though slight modifications of the gingival zeniths of the centrals and laterals were deemed, the patient's unwillingness to undergo any sort of surgical procedure prompted it to be unaltered. Preparation of the anterior abutments were carried out and checked through indices such as to achieve correct amount of depth of reductions in order to avoid over contoured final restorations [Figure 4], final impressions were made after necessary gingival retraction, casts poured, subsequent face bow transfer done and casts were articulated through centric relation records. Putty indices made from the desired wax ups were used to fabricate the provisionals and necessary slight intra oral adjustments were made and patient was instructed to report back after $2-3$ weeks. A pleasing and desirable esthetics was achieved with the provisionals [Figure 5]. Subsequent follow ups when revealed desirable gingival contours and no damage to the provisionals, definitive restorations were planned. Wax up followed by metal trial, shade matching was performed with the help of vita 3D master shade guide and subsequent veneering of porcelain for ceramo metallic restorations [Figure 6]. The palatal surfaces of the maxillary anterior restorations were finished in metal in order to provide stable centric contacts and a smooth unhindered anterior guidance without any alteration of vertical dimensions. The restorations were checked for fit, esthetics and proper margins. Intra oral adjustments were done and the restorations were luted [Figure 7a \& 7b]. The patient was recalled and was evaluated after a week's interval.

\section{Discussion:-}

The anterior guidance is a coupled result of both anterior tooth position and border movements. The guidelines followed for long-term stability are: Disclusion of all posterior teeth on protrusion; balancing side; stable centric holding contacts and position and contour of anterior teeth in harmony with the lip closure pathway and envelope of function. ${ }^{3}$

This case report humbly portrayed all these necessary considerations for preservation of the anterior guidance, as the plausible rationale being that the patient produced disclusion in excursive movements which is the desired occlusion scheme in periodontal healthy dentition as compared to a group function occlusion which would have prompted us to alter the guidance to achieve disclusion. A slight attritional wear on mandibular anterior teeth was probably due to be deflective incline interferences to centric relation. If the incisal guidance would have been altered it would have led to combined reduction of both overjet and overbite, thereby concentrating much stress in the incisal edge area with possible chipping and fracture of the margins. By preserving, desired amount of disclusion was achieved through a canine guided scheme thus the mutual protection of teeth as well as restorations was in harmony [Figures 8 a -c \& 9]. Further finishing of the palatal surfaces of the maxillary teeth in metal provided firm, stable centric contacts despite the rise and belligerent use of all ceramic more aesthetic materials justifies the importance of anterior guidance. The aesthetic treatment also involved reestablishment of correct incisal arching in consonance with contour of the lower lip by restoring adequate shape and proportion, and placing the maxillary incisal edges at the wet and dry junctions of the mucosa of the lower lip thus additively ameliorating the aspect of phonetics [Figures $10 \mathrm{a} \& 10 \mathrm{~b}$. The need of the hour in recent times facilitates use of digital software programs for esthetic makeovers but not all the programs available today provide the same competency for comprehensive analysis and other factors such as ease of use, cost, time efficiency, and compatibility of the program with CAD/CAM ${ }^{4}$ or other digital systems often promotes the clinician to take a more conventional and economic approach for the benefit of the patients.

\section{Conflicting Interest: (If present, give more details):} Nil 
Figures And Legends :

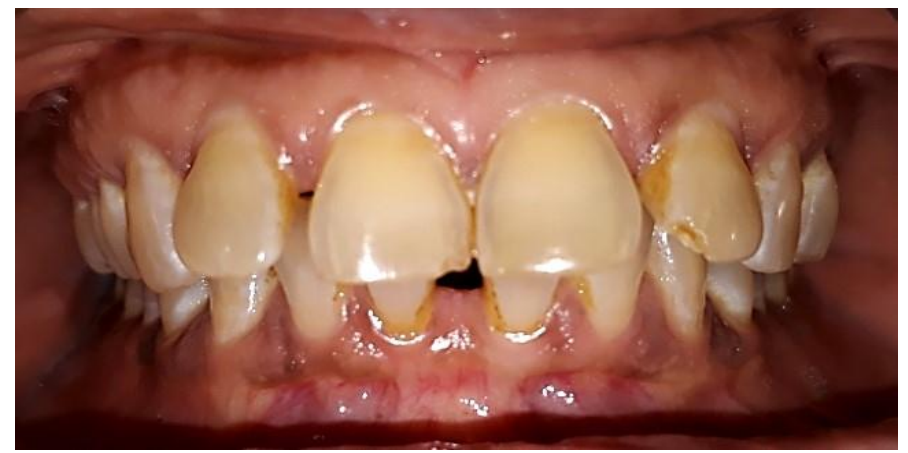

Figure 1:- Pre-treatment intra oral frontal view.

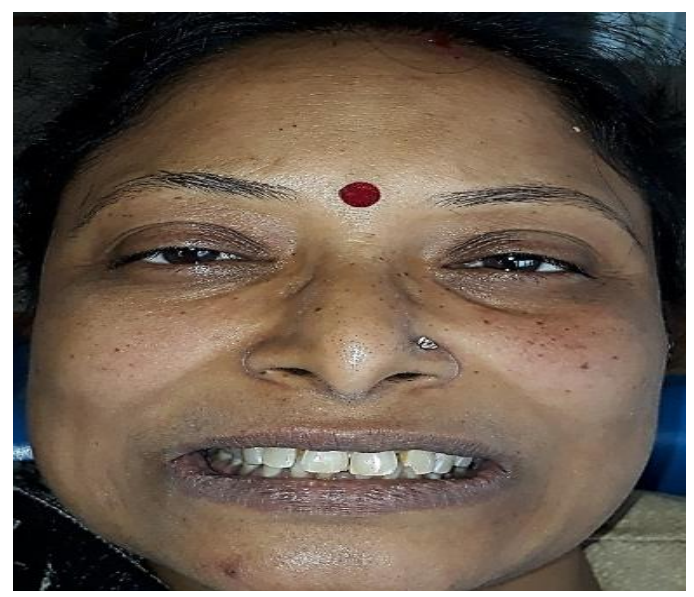

Figure 2:- Pre-treatment Extra oral frontal view.

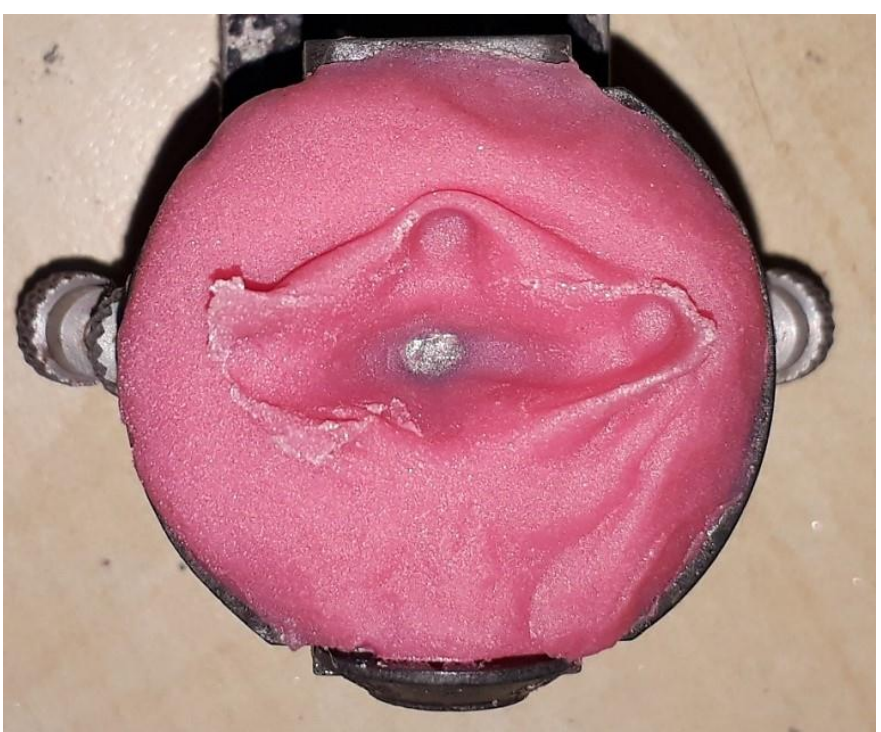

Figure 3:- Customization of anterior guidance. 


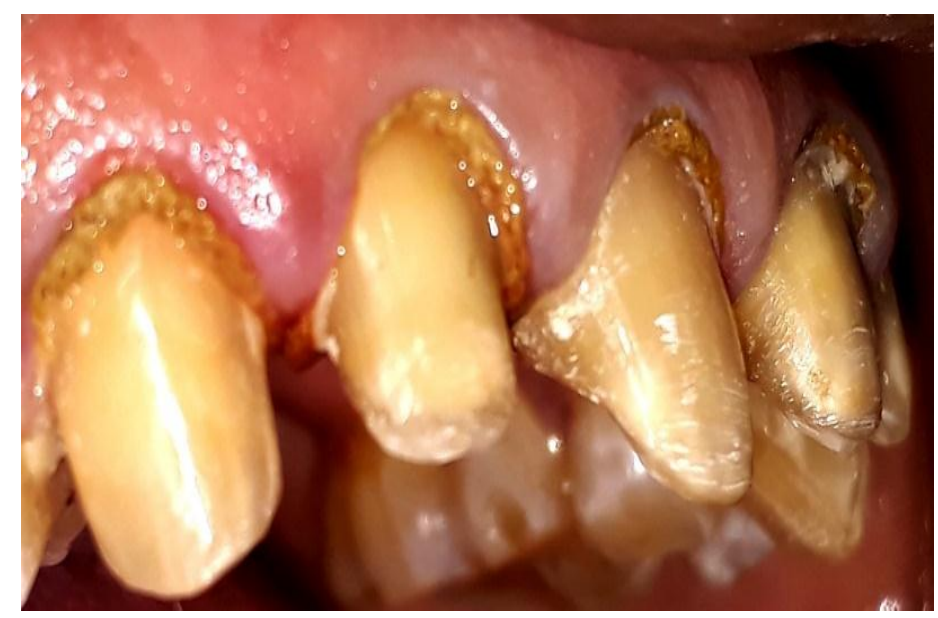

Figure 4:- Tooth preparation.

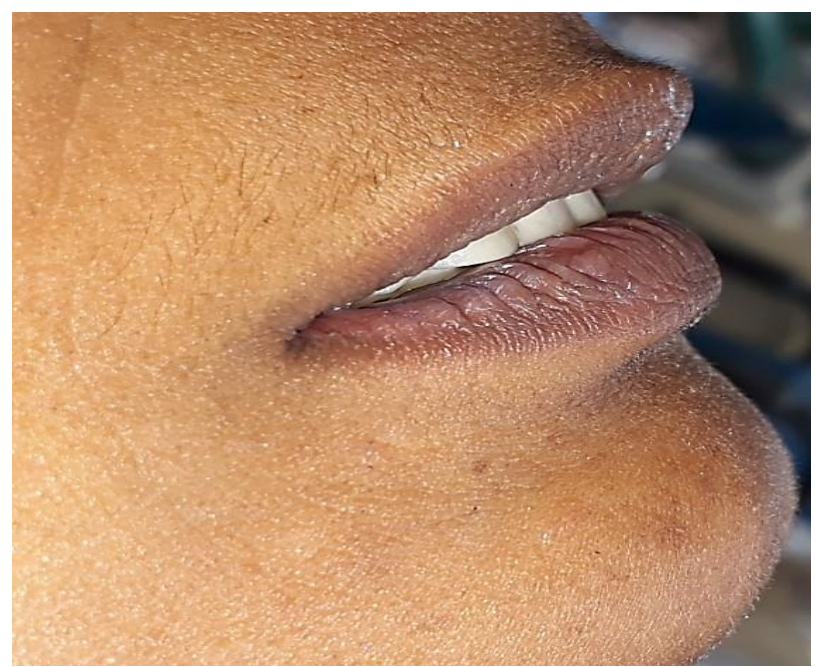

Figure 5:- Provisional restorations.

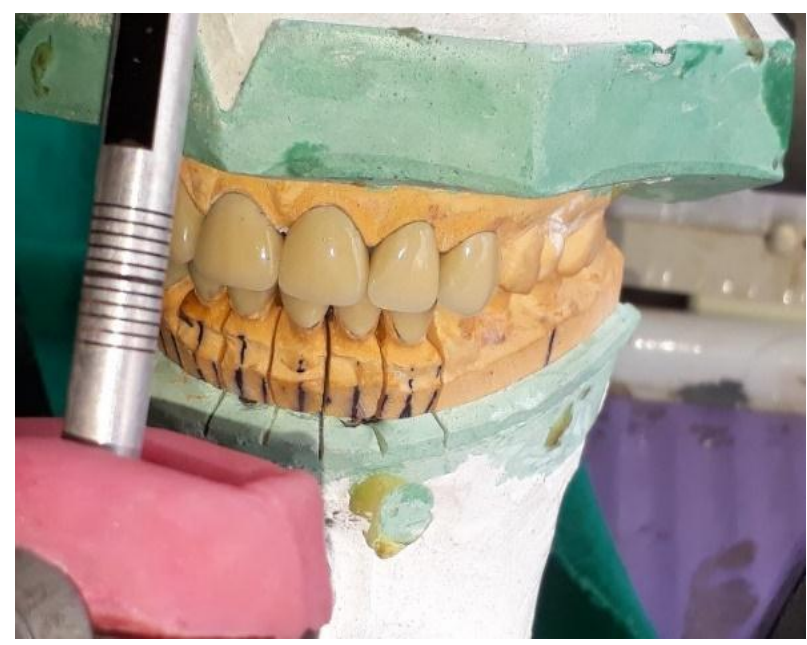

Figure 6:- Finished restorations according to customized anterior guidance. 


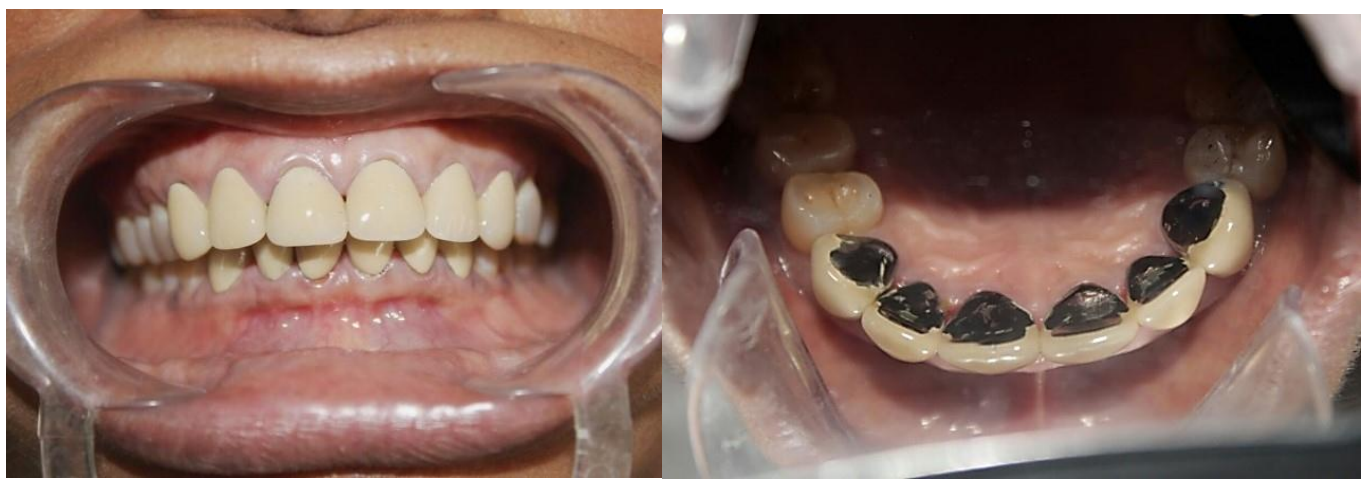

Figure 7a \&7b:- Intra oral frontal view and Occlusal view of Final restorations.

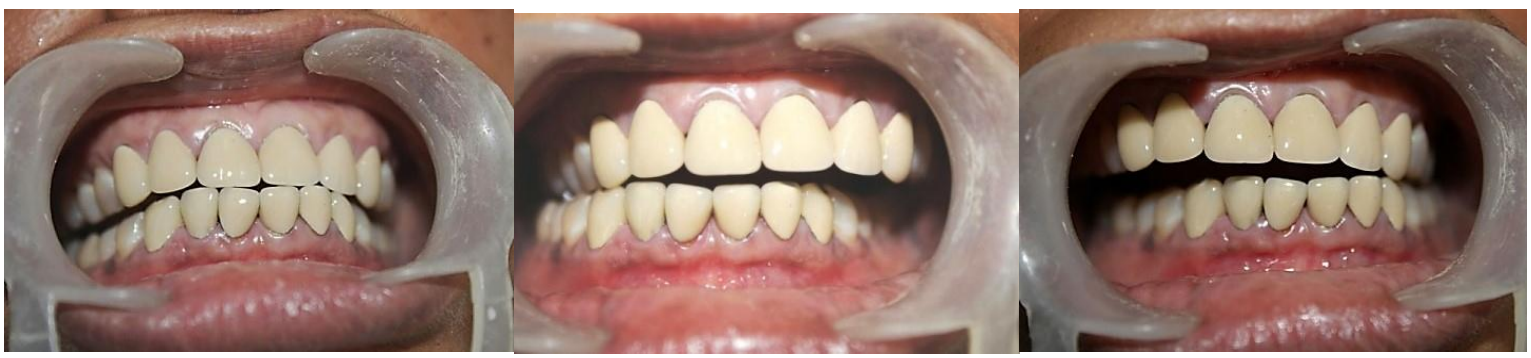

Figure 8a:- Disclusion achieved in protrusive, right and left excursive movements.

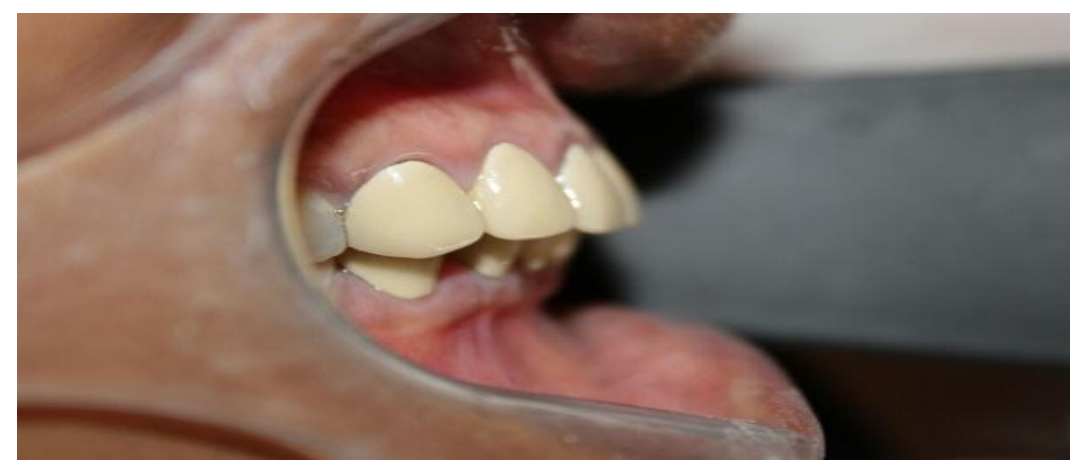

Figure 9: Post op lateral view.

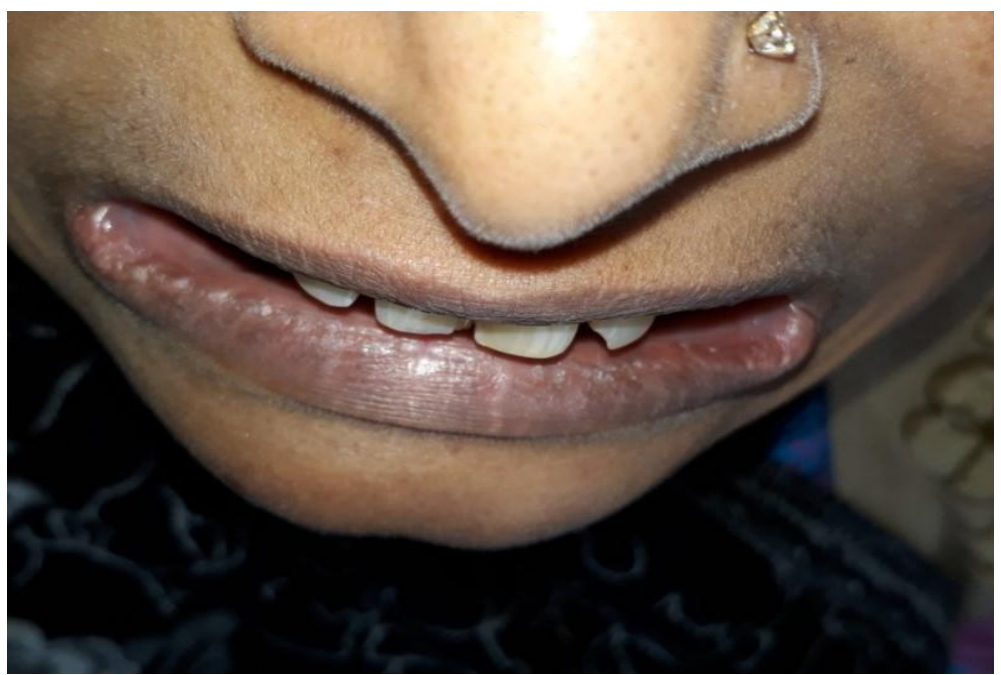

Figure 10a:- Pre-treatment extra oral frontal view depicting straining of lips and unpleasant smile. 


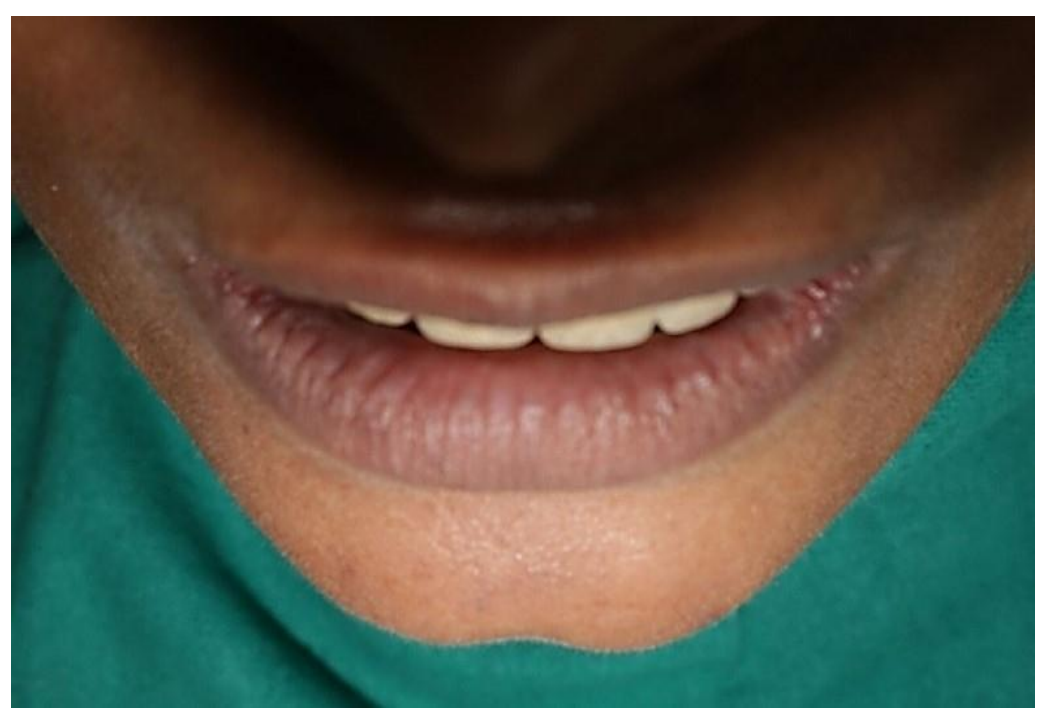

Figure 10b:- Post treatment extra oral frontal view showing correct incisal contouring and pleasant smile.

\section{References:-}

1. Broderson SP. Anterior guidance - the key to successful occlusal treatment. J Prosthet Dent 1978;39:396-400.

2. Re GJ, Nelson SJ. Custom incisal guide table fabrication. J Prosthet Dent 1997;77:454.

3. Dawson PE. Evaluation, diagnosis and treatment of occlusal problems. St. Louis: Mosby; 1974.

4. Omar D, Duarte C. The application of parameters for comprehensive smile esthetics by digital smile design programs: A review of literature. Saudi Dental J 2018; 30:7-12. 